\title{
Mesenchymal stem cell treatment for refractory pediatric rheumatic diseases: A single center case series
}

\section{Stephen Chee-Yung Wong}

University of Washington https://orcid.org/0000-0003-1617-9634

\section{Leah C Medrano}

University of California Los Angeles

\section{Alice D Hoftman}

University of California Los Angeles

Olcay Y Jones

Walter Reed National Military Medical Center

Deborah K McCurdy ( $\nabla$ dmccurdy@mednet.ucla.edu )

University of California Los Angeles

\section{Case Report}

Keywords: Mesenchymal, Stem cell, Lupus, Arthritis, Treatment, Novel, Pediatric, Refractory

Posted Date: July 15th, 2020

DOI: https://doi.org/10.21203/rs.3.rs-41616/v1

License: (c) (i) This work is licensed under a Creative Commons Attribution 4.0 International License. Read Full License 


\section{Abstract}

\section{Background:}

Pediatric rheumatic disease treatment has seen a surge in innovative therapies including numerous biologic medications that show great potential. Yet, many children with advanced disease are not able to reach remission on available treatment. Mesenchymal stem cells have immunomodulatory properties, and transplantation of these stem cells have been used to successfully treat immunologic conditions like graft-versus-host disease. Mesenchymal stem cell research in adult lupus has been encouraging, but there is a lack of studies in pediatric rheumatology. This retrospective case series will highlight three children with refractory autoimmune disease who received mesenchymal stem cell therapy and review the potential benefits and risks of this emerging treatment modality.

\section{Case Presentation:}

Three children with established diagnoses of systemic lupus erythematosus, mixed connective tissue disease and juvenile idiopathic arthritis were under the care of pediatric rheumatology at a large, tertiarycare, teaching institution for many years. Multiple non-biologic and biologic disease-modifying antirheumatic drugs failed to significantly decrease disease activity, and as a result, the families chose to undergo mesenchymal stem cell therapy. After transplantation, all children improved and were able to taper off conventional immunosuppressive drugs. No serious adverse events occurred.

\section{Conclusion:}

The three cases presented in this study reflect comparable beneficial outcomes and minimal risks published in adult studies. Mesenchymal stem cell transplantation is a promising adjunctive treatment option that necessitates further research and development of standardized infusion therapy protocols.

\section{Background}

The treatment of pediatric rheumatic diseases has advanced greatly over past two decades with the discovery and widespread use of biologics, allowing improved disease control in many cases. However, a significant number of patients, including those with systemic lupus erythematosus (SLE), mixed connective tissue disease (MCTD), and juvenile idiopathic arthritis (JIA), continue to have significant disease activity despite maximized usage of conventional therapeutic agents. Further investigations are needed for exploring new treatment modalities to improve patient outcomes.

Mesenchymal stem cell (MSC) transplantation has been used to treat steroid-refractory graft-versus-host disease (GVHD). MSCs, also known as medicinal signaling cells, have been studied for potential efficacy in many different illnesses ranging from multiple sclerosis to myocardial infarction with promising results [1]. The theoretical properties for how MSCs can ameliorate certain diseases derive from its known immunomodulatory actions including suppressing $T$ cell proliferation, B cell activation, and inflammatory 
cytokine production [2-4]. With these anti-inflammatory and immunosuppressive properties in mind, researchers have investigated how MSC treatment would affect the course of SLE in certain adult populations with promising results [5-7]. Since MSC research remains a political, cultural, and religious area of contention, the breadth of research, especially in the pediatric population, is scarce within the United States. In this retrospective case series, we present our experience of MSC treatment in three patients who independently undertook this treatment after second opinion visits to commercial clinics licensed in the US or abroad.

\section{Case Presentation}

\section{Case 1}

A 16-year-old female with SLE initially presented at the age of 9 years with the sole symptom of idiopathic scleritis requiring steroid eye drops intermittently for flares. After 3 years into her illness, she developed polymyositis with severe arthritis with elevated muscle enzymes, speckled antinuclear antibody (ANA) of 1:640, and negative lupus serologies for double stranded (ds)DNA and extractable nuclear antigen (ENA). She was diagnosed with undifferentiated connective tissue disease (UCTD) and started on methotrexate and meloxicam with minimal efficacy.

In the next nine months, she progressed to develop a malar rash and sustained leukopenia and lymphopenia. Her diagnosis was subsequently refined to SLE, meeting American College of Rheumatology 1997 revised criteria with malar rash, arthritis, positive ANA, and hematologic abnormalities. Her treatment included hydroxychloroquine and prednisone followed by disease-modifying anti-rheumatic drugs (DMARDs) and biologics as steroid-sparing medications including methotrexate, leflunomide, abatacept, tocilizumab and azathioprine over the following 48 months with limited efficacy. She continued to have recurrent scleritis and active arthritis causing significant pain.

After consultation with a company in Texas, Celltex, the patient and her family made the decision to travel to Cancun, Mexico for autologous adipose tissue-derived MSC transplantation. The cells were harvested from abdominal adipose tissue by liposuction in California, sent to Celltex for MSC isolation and preparation for transplant that was infused in a clinic in Cancun, Mexico. She received one intranasal injection $\left(100 \times 10^{6}\right.$ cells), one lymph node injection $\left(100 \times 10^{6}\right.$ cells), three intravenous transfusions (379 $\times 10^{6}, 256 \times 10^{6}$, and $393 \times 10^{6}$ cells) given within one week, then one further intravenous transfusion $\left(234 \times 10^{6}\right.$ cells $)$ nine months later. The target dose per treatment was $200 \times 10^{6}$ cells $\left(3.3 \times 10^{6}\right.$ MSCs $/ \mathrm{kg} / \mathrm{dose}$ ). While she was on MSC treatment, all conventional immunosuppressive medications were discontinued. After receiving MSC transplantation, she had a transient flare with arthralgia, malar rash, and recurrence of scleritis, which subsequently resolved in 2 months without treatment. Otherwise, she had no other minor or serious adverse effect from MSC transplantation. During 18 months follow-up since MSC, she remains clinically stable; patient global assessment (PGA) decreased from 8/10 to 1/10 after treatment and physician global assessment from 7/10 to 2/10. The ANA titer declined from 1:640 to 1:80-160. Currently, she is only on intermittent topical ocular prednisolone therapy for rare patient- 
reported scleritis episodes and continues to have regular visits to pediatric rheumatology and ophthalmology clinics for routine monitoring.

\section{Case 2}

A 19-year-old female was diagnosed with MCTD consisting of SLE and systemic sclerosis features at age of 15 years. Initial presentation included intermittent fevers, Raynaud's phenomenon, cervical lymphadenopathy, arthritis in her wrist and hips, generalized fatigue, weakness, dyspnea, and photosensitivity. Laboratory evaluation revealed high titer ANA ( $>1: 1280$, Speckled), positive serologies for dsDNA and ribonucleoprotein antibodies and positive rheumatoid factor (RF). Ultrasound-guided lymph node biopsy showed benign reactive lymphadenopathy; no malignant cells or infectious organisms were identified. She was treated with multiple DMARDs and biologics including methotrexate, hydroxychloroquine, leflunomide, mycophenolate mofetil, rituximab, abatacept, and belimumab. She remained on intermittent low to moderate dose oral prednisone throughout this time. Her response to treatment was marginal; her annual pulmonary function studies showed gradual reduction in diffusing capacity for carbon monoxide (DLCO) down to $41.5 \%$ predicted, although her high-resolution chest computed tomography and repeat echocardiograms remained normal.

Three years into her treatment, she developed significant pain and weakness. She was admitted and treated for polymyositis. Magnetic resonance imaging (MRI) revealed avascular necrosis of both hips. She was started on tocilizumab and received four doses administered intravenously every 14 days with improvement. Due to poor quality of life from hip pain, she pursued MSC treatment locally at AMA Regenerative Medicine \& Skincare. The MSC were derived from Wharton's jelly after a cesarean birth and processed under a strict procedure by Vitti Labs (a Food and Drug Administration-registered tissue bank) to ensure viability, non-infectivity, and lack of genetic mutations. She received MSCs via intravenous transfusion as well as intraosseous injections directly into the head of the femurs bilaterally. Three hip injections ( $1 \mathrm{cc}$ of Wharton's Jelly MSC; $1.1 \times 10^{6} \mathrm{MSC}$ ) were given at months 0 , 2 , and 6 , while intravenous transfusions ( $\left.60 \times 10^{6} \mathrm{MSCs} ; 1.2 \times 10^{6} \mathrm{MSCs} / \mathrm{kg} / \mathrm{dose}\right)$ were given at months 2 and 6 . She continued on twice monthly intravenous tocilizumab during the first 2 months of MSC treatment; all conventional immunomodulatory therapy was discontinued thereafter. The patient reported fatigue and local pain at the site of the hip injections for three days but did not have any minor or serious adverse effect. Patient reported an improvement with a pre-treatment PGA of $7 / 10$ and post-treatment PGA of $2 / 10$. She had previously dropped out of her college semester pre-transplant and was able to return to full time studies and dance. Although her serologies remained unchanged, the erythrocyte sedimentation rate (ESR) normalized for 3 months after initial MSC therapy and remains only mildly elevated compared to pre-treatment levels. Although there continues to be radiographic evidence of avascular necrosis of the hips, there has been no collapse of the femoral head and the scheduled bilateral hip replacement surgery has been cancelled.

\section{Case 3}


An 18-year-old female with diagnosis of RF positive and HLA B27 positive JIA presented initially at 11 years of age with right knee arthritis, then progressed to arthritis in bilateral knees, left elbow, and cervical spine. Her arthritis activity correlated with increased ESR. Treatment included non-steroidal antiinflammatory drugs (naproxen, sulindac, meloxicam), followed by methotrexate (initially oral, then subcutaneous). Incidentally, she also had thoracic outlet syndrome causing neck pain and right arm paresthesia during dirt bike racing. For two years, she transferred her care to another institution, where she began etanercept with methotrexate. She had a brief remission three years into her initial diagnosis. A routine MRI of her elbow then showed active synovitis and new erosions, which led to a recommendation to switch to another biologic therapy. However, severe localized pain with biologic injections prompted the family to explore stem cell therapy abroad. She transferred back to our institution due to disagreement about MSC therapy with the outside institution. Her PGA prior to MSC treatment was 6/10.

She underwent an umbilical cord MSC transplant at Stem Cell Institute in Panama at the age of 16 years old. The transplant involved three intravenous infusions over four days with cells derived from donor human umbilical cord tissue-derived MSC $\left(120 \times 10^{6} \mathrm{MSCs} ; 1.8 \times 10^{6} \mathrm{MSCs} / \mathrm{kg} / \mathrm{dose}\right)$. The transplant physicians instructed the patient to wean off her rheumatic medications prior to the transfusion. She had no initial side effects, though developed a migratory arthritis flare 5 weeks post-transplantation, that was attributed to a Jarisch-Herxheimer reaction. No other minor or serious adverse effects were reported.

An MRI of her left elbow 5 months post-transplantation revealed continued active synovitis and progression of erosive changes. Post-transplantation labs remained unchanged with positive RF, as well as positive anti-cyclic citrullinated peptide antibodies, but there was a normalization of ESR. A biologic medication was prescribed but never taken by the patient. She was maintained on celecoxib therapy in the interim. One year after her initial transplant, she returned to Panama for a second MSC intravenous transfusion $\left(120.6 \times 10^{6}\right.$ cells; $1.9 \times 10^{6} \mathrm{MSCs} / \mathrm{kg} /$ dose) . She developed flu-like symptoms with this treatment. Follow-up 3 months later showed worsening elbow contracture and active synovitis on MRI, though no worsening of erosions. She had an intra-articular steroid injection to the elbow joint the following month with marked improvement in her symptoms. Family decided to initiate a complement of holistic management including monthly hyperbaric oxygen, red-light therapy with in-home lymphatic draining twice weekly, and daily pulsed electromagnetic field therapy. Her most recent PGA is $1 / 10$ and the family is content with her current condition.

\section{Discussion And Conclusions}

All three cases presented in this report demonstrated years of poorly controlled disease unresponsive to multiple non-biologic and biologic DMARDs prior to initiating MSC treatment. None had serious adverse effects such as infection, emboli, ectopic growth, or malignancy. Although some mild adverse effects were endorsed, all patients felt that MSC treatment was beneficial in achieving disease remission with drastic improvement in patient global assessment. It should be remarked that disease remission may be confounded by conjunctive treatment as seen in case 2 with co-administration of MSC with tocilizumab, and in case 3 with an intra-articular steroid injection after MSC treatment. In addition to clinical 
improvement, laboratory changes revealed normalization of ESR, although specific autoantibody titers did not change.

There are many similarities that become apparent when comparing these three patients who received MSCs, although each patient had unrelated pediatric rheumatic diseases. The perceived benign potential benefit of MSC and how they can modulate the immune response led families to choose this experimental and adjunctive treatment regimen for their children. The decisions to adopt MSC treatment was difficult since stem cell research is lacking in the field of pediatric rheumatology. None of these patients nor their families were recommended by their care team providers to pursue MSC therapy; the decision was solely made by the family. All families paid for MSC treatment out-of-pocket; current treatment is very expensive and averages in the tens of thousands of dollars per transfusion. One family sold their car, and a second family re-mortgaged their home in order to pay for the treatment they felt was necessary to alleviate their child's rheumatic disease.

MSCs are multipotent progenitor cells that can differentiate into various cell lines, including myocytes, adipocytes, chondrocytes, and osteoblasts. They were first hypothesized in the late nineteenth century but was formally discovered in the 1970s by Alexander Friedenstein [8-9]. In 1991, Arnold Caplan first named these cells as MSCs [10]. Historically, MSCs have been isolated from cell cultures, but their native residence in vivo remained unknown for many years. It was not until the revelation of vascular pericytes (cells that enclose small blood vessels like capillaries) and their expressed MSC markers CD73, CD90 and CD105 that MSCs were discovered in vivo [11-12]. MSCs are harvested from donors (allogeneic) or from the patient (autologous), mostly from bone marrow, adipose tissue, and placental/umbilical cord. Tissue banks and harvesting facilities have specific criteria and procedures for procurement of MSCs for clinical use, but no universal guidelines for the manufacturing process exist, leading to heterogenous MSC yields [13].

MSC transplantation is a very specialized treatment modality with which few rheumatologists feel comfortable utilizing. Regulating authorities globally have investigated its potential for treatment in a whole host of diseases. Canada, New Zealand, and some European Union countries have approved Prochymal ${ }^{\circledR}$, while in Japan, TEMCELL ${ }^{\circledR}$ HS Inj. has been approved for GVHD [14]. In the United States, MSC have been conferred orphan drug designation by the US Food and Drug Administration but has not been fully approved. Furthermore, no standard dosing has been published for pediatric rheumatic disease. There have been a few studies investigating the clinical efficacy and safety of MSC treatment in adult SLE with intravenous doses ranging from $1 \times 10^{6} \mathrm{MSCs} / \mathrm{kg} / \mathrm{dose}$ to $2 \times 10^{8} \mathrm{MSCs} / \mathrm{kg} / \mathrm{dose}$ [15]. In this case series, the patients' dosing ranged from $1.2 \times 10^{6} \mathrm{MSCs} / \mathrm{kg} /$ dose to $6.5 \times 10^{6} \mathrm{MSCs} / \mathrm{kg} / \mathrm{dose}$. In contrast, recommended dosing for MSC therapy in acute GVHD is $1 \times 10^{6} \mathrm{MSCs} / \mathrm{kg} / \mathrm{dose}$ to $2 \times 10^{6}$ MSCs $/ \mathrm{kg} /$ dose weekly for a total of 4 doses. Studies have shown that providers do not tightly adhere to recommended dosing of GVHD with an average dose of $1.4 \times 10^{6} \mathrm{MSCs} / \mathrm{kg}$ and three total doses administered (range is 1-10 doses administered) [16]. With such varying doses in MSC treatments, adverse events might be expected in the comparatively higher-dosed infusion; but overall, studies have not shown significant adverse events, which is consistent with our observations [15-16]. There has been 
some concern for tumorigenic potential of MSCs, but a recently published systematic review did not find this correlation to be significant; no serious adverse events were reported in this study [17]. Although MSC transplantation is generally considered safe, further research and reporting of adverse events will assist pediatric rheumatologists in weighing the risks and benefits of this new treatment modality. Standardized procurement, preparation, cell viability, the number of cell cycles, and treatment protocols should be established for pediatric rheumatic disease.

This case series demonstrates variability in the utilization of MSCs transplantation in the source of cells, types of cells, and route of administration. Two patients received allogeneic umbilical tissue-derived MSCs, while one patient received autologous adipose tissue-derived MSCs. Recent research has suggested that allogeneic MSC transplantation may be more immunosuppressive than autologous MSCs. MSCs derived from lupus patients have shown dysfunction in both proliferation and immunoregulation and appear to be phenotypically senescent [18]. All patients received systemic treatment intravenously, and two patients also received local injection (bone, lymph node, and intranasal). Local administration leads to increased complexity of differentiated engrafted MSCs due to microenvironmental milieu. On the other hand, systemic treatment allows infused MSCs to traffic to injured and inflamed tissues through chemokines and growth factors [19]. Dosing intervals in our series ranged from monthly to yearly infusions. Since there has been a scarcity of MSC research in pediatric rheumatology, protocol variability is to be expected. With an increase of adult MSC treatment publications in the past few years, more families will continue to seek this emerging therapy for their children with refractory disease. This study aims to bring awareness of this novel treatment to the pediatric rheumatology community. Although stem cell therapy remains controversial, especially in the United States, ongoing research and consideration of MSC therapy in refractory pediatric rheumatic disease may be a fruitful endeavor.

This retrospective series highlights the potential of mesenchymal stem cell therapy in refractory pediatric rheumatic diseases. Clinical improvement was observed in our small patient cohort. Despite the multiple barriers that patients and their families must overcome, the immunomodulatory effects of MSC treatment on autoimmune inflammatory disease makes it a promising adjunctive treatment. However, we strongly believe this treatment modality remains poorly studied and should be cautiously approached with structured trials in a formal clinical setting under guidance of experienced clinician-scientists familiar with this specialized therapy. Further evaluation of the biological properties, clinical safety, and efficacy of MSC therapy in specific pediatric rheumatic diseases is warranted to inform clinical practice.

\section{Abbreviations}

SLE

Systemic lupus erythematosus

MCTD

Mixed connective tissue disease

JIA 
Juvenile idiopathic arthritis

MSC

Mesenchymal stem cell

GVHD

Graft-versus-host disease

ANA

Antinuclear antibody

dsDNA

Double stranded deoxyribonucleic acid

ENA

Extractable nuclear antigen

UCTD

Undifferentiated connective tissue disease

DMARDs

Disease-modifying anti-rheumatic drugs

PGA

Patient global assessment

RF

Rheumatoid factor

MRI

Magnetic resonance imaging

ESR

Erythrocyte sedimentation rate

HLA

Human leukocyte antigen

\section{Declarations}

\section{Ethics approval and consent to participate}

University of California Los Angeles Institutional Review Board waived the requirement of ethics and consent approval due to nature of the study. The views expressed in this article are those of the author (OYJ) and do not reflect the official policy of the Department of Army/Navy/Air Force, Department of Defense, or U.S. Government.

\section{Consent for publication}

Verbal and written consent for publication was obtained from the patient, or in the case of children, their parent or legal guardian. All clinics and companies mentioned consented to being named in this study. 
Availability of data and materials

Data sharing is not applicable to this article as no datasets were generated or analyzed during the current study. Any case details used in the current study are available from the corresponding author upon reasonable request.

\section{Competing interests}

SCW, LCM, ADH, OYJ, DKM - The authors declare that they have no competing interests or financial relationships relevant to this article to disclose.

\section{Funding}

No direct or indirect funding was secured or utilized in the development of this study/manuscript.

\section{Authors' contributions}

SCW conceptualized, gathered clinical data, analyzed, interpreted, drafted the initial manuscript, reviewed and revised the manuscript; LCM gathered clinical data, analyzed, interpreted, drafted the initial manuscript, and reviewed the manuscript; $A D H$ conceptualized, reviewed and revised the manuscript; OYJ conceptualized, reviewed, and revised the manuscript; DKM conceptualized, reviewed and revised the manuscript. All authors approved the final manuscript as submitted and agreed to be accountable for all aspects of the work.

\section{Acknowledgements}

We express our appreciations to the patients, families, clinics, and tissue banks for sharing their experience and access to specifics of stem cell treatment protocols used.

\section{References}

1. Kim N, Cho S. Clinical applications of mesenchymal stem cells. Korean J Intern Med. 2013;28(4):387-402.

2. Di Nicola M, Carlo-Stella C, Magni M, et al. Human bone marrow stromal cells suppress T-lymphocyte proliferation induced by cellular or nonspecific mitogenic stimuli. Blood. 2002;99:3838-43.

3. Spaggiari GM, Capobianco A, Abdelrazik H, Becchetti F, Mingari MC, Moretta L. Mesenchymal stem cells inhibit natural killer-cell proliferation, cytotoxicity, and cytokine production: role of indoleamine 2,3-dioxygenase and prostaglandin E2. Blood. 2008;111:1327-33.

4. Corcione A, Benvenuto F, Ferretti $E$, et al. Human mesenchymal stem cells modulate B-cell functions. Blood. 2006;107:367-72. 
5. Liang $\mathrm{J}$, Zhang $\mathrm{H}$, Hua B, et al. Allogenic mesenchymal stem cells transplantation in refractory systemic lupus erythematosus: a pilot clinical study. Ann Rheum Dis. 2010;69:1423-9.

6. Sun L, Wang D, Liang J, et al. Umbilical cord mesenchymal stem cell transplantation in severe and refractory systemic lupus erythematosus. Arthritis Rheum. 2010;62:2467-75.

7. Liang J, Gu F, Wang H, et al. Mesenchymal stem cell transplantation for diffuse alveolar hemorrhage in SLE. Nat Rev Rheumatol. 2010;6:486-9.

8. Cohnheim J. Ueber Entzündung und Eiterung. Archiv Pathologische Anatomie Physiologie Klinische Medicin. 1867;40:1-79.

9. Friedenstein AJ, Chailakhyan RK, Latsinik NV, Panasyuk AF, Keiliss-Borok IV. Stromal cells responsible for transferring the microenvironment of the hemopoietic tissues. Cloning in vitro and retransplantation in vivo. Transplantation. 1974;17:331-40.

10. Caplan Al. Mesenchymal stem cells. J Orthop Res. 1991;9:641-50.

11. Crisan M, Yap S, Casteilla L, Chen CW, Corselli M, Park TS, et al. A perivascular origin for mesenchymal stem cells in multiple human organs. Cell Stem Cell. 2008;3:301-13.

12. Corselli M, Chen CW, Sun B, Yap S, Rubin JP, Peault B. The tunica adventitia of human arteries and veins as a source of mesenchymal stem cells. Stem Cells Dev. 2012;21:1299-308. doi:10.1089/scd.2011.0200.

13. Phinney DG, et al. Manufacturing mesenchymal stromal cells for clinical applications: A survey of Good Manufacturing Practices at U.S. academic centers. Cytotherapy. 2019;21:782-92.

14. Galipeau J, Sensébé L. Mesenchymal Stromal Cells: Clinical Challenges and Therapeutic Opportunities. Cell Stem Cell. 2018;22:824-33.

15. Zhou T, Li HY, Liao C, Lin W, Lin S. Clinical Efficacy and Safety of Mesenchymal Stem Cells for Systemic Lupus Erythematosus. Stem Cells Int. 2020. doi:10.1155/2020/6518508.

16. Elgaz S, Kuçi Z, Kuçi S, Bönig H, Bader P. Clinical Use of Mesenchymal Stromal Cells in the Treatment of Acute Graft-versus-Host Disease. Transfus Med Hemother. 2019;46:27-34.

17. Lalu MM, McIntyre L, Pugliese C, Fergusson D, Winston BW, et al. Safety of Cell Therapy with Mesenchymal Stromal Cells (SafeCell): A Systematic Review and Meta-Analysis of Clinical Trials. PLoS ONE. 2012;7:e47559. doi:10.1371/journal.pone.0047559.

18. Cheng R-J, Xiong A-J, Li Y-H, Pan S-Y, Zhang Q-P, Zhao Y, Liu Y, Marion TN. Mesenchymal Stem Cells: Allogeneic MSC May Be Immunosuppressive but Autologous MSC Are Dysfunctional in Lupus Patients. Front Cell Dev Biol. 2019;7:285. doi:10.3389/fcell.2019.00285.

19. Ra JC, Kang SK, Shin IS, et al. Stem cell treatment for patients with autoimmune disease by systemic infusion of culture-expanded autologous adipose tissue derived mesenchymal stem cells. J Transl Med. 2011;9:181. doi:10.1186/1479-5876-9-181. 